\title{
Importance of Studying and Discussing the Management of Complications During Neurosurgical Procedures
}

\author{
Homajoun Maslehaty and Athanasios K Petridis*
}

Department of Neurosurgery, Klinikum Duisburg, Academic Teaching Hospital of University Essen-Duisburg, Germany

The neurosurgical training is one of the most demanding ones. Studying and mastering the anatomy of the human central nervous system, reading and understanding scientific papers, learn how to perform surgeries, which can bring the surgeon to its limits day for day, are three equal columns in the making of a good neurosurgeon.

Except of studying surgeries and books and learning from grand masters the study of some ones own surgeries and own mistakes become more and more important. Video recording of the surgeries and identifying the possible mistakes are very effective in order to correct future mistakes. Which step has led to a complication and how the complication was managed in a "self-made" video record proved, at least for us, to be a very effective way to improve surgical skills.

On the other hand it is also important to be able to confess own mistakes and complications and openly discuss them with others. Every single active surgeon has produced complications and had to deal with them. Discussing the strategies to manage such complications is highly valued.

As an example we demonstrate a recorded surgical video of a patient we have operated on a recurrent lumbar disc herniation. First surgery was about 2 years ago. The tissue was scarified and scar was fused with dura. We started recording the surgery at the moment of cutting the thoraco-dorsal fascia. Dissection of the dura and freeing it from scar tissue seemed to be easy. At one point though we applied to much traction on the scar tissue and the dura was injured. Everything was kept on the recorded video. We proceeded with the dissection of the dura in another region, away from the injured dura. Freeing the dura from scar tissue allowed us to oversee the dural leakage and to apply an allograft sealing. CSF leakage stopped and surgery was proceeded as usual, performing the removal of the disc hernia. After sequestrectomy the dura sealing material was removed and the dural injury was sutured by just one stitch. Valsalva manoeuvre showed no further CSF leakage. Additionally the dura was sealed again with allogenic material and application of fibrin glue.

After surgery the patient had no additional complications and was discharged 5 days after surgery. We studied the surgical video to memorize when exactly the dural injury took place and how we did proceed. Was there anything we could do different? Watching the surgery in a calm environment without intraoperative stress allows review of surgery in a much more effective way and to observe how the different steps had been performed and in addition how they could be optimized.

By sharing experiences of surgical complications will help the neurosurgical trainees equally as the neurosurgical expert to study and learn from others.

In a special issue on neurosurgical complications we invite neurosurgeons to share their experiences with intraoperative complications and discuss how they managed such complications. The experience of every one will become the experience of all of us and will allow us to manage complications more effectively.
${ }^{*}$ Corresponding author: Athanasios $\mathrm{K}$ Petridis, Associate Professor of Neurosurgery, Neurosurgical Department, Wedau Kliniken, Sana Konzern, Zu den Rehwiesen 947055 Duisburg, Germany, Tel: +49-203-733-0; Fax: +49-203-7330; E-mail: opticdisc@aol.com

Received June 20, 2013; Accepted June 20, 2013; Published June 22, 2013

Citation: Maslehaty H, Petridis AK (2013) Importance of Studying and Discussing the Management of Complications During Neurosurgical Procedures. J Neurol Disord 2: e103. doi:10.4172/2329-6895.1000e103

Copyright: (C) 2013 Maslehaty $\mathrm{H}$, et al. This is an open-access article distributed under the terms of the Creative Commons Attribution License, which permits unrestricted use, distribution, and reproduction in any medium, provided the original author and source are credited. 
Citation: Maslehaty H, Petridis AK (2013) Importance of Studying and Discussing the Management of Complications During Neurosurgical Procedures. J Neurol Disord 2: e103. doi:10.4172/2329-6895.1000e103

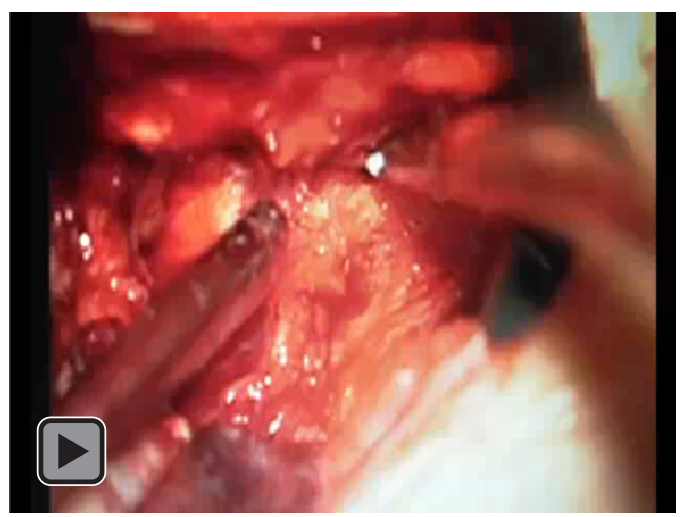

Video 1: During dissection of the dura epidural scar tissue is removed Because of traction on the scar the dura is damaged and CSF leakage started.

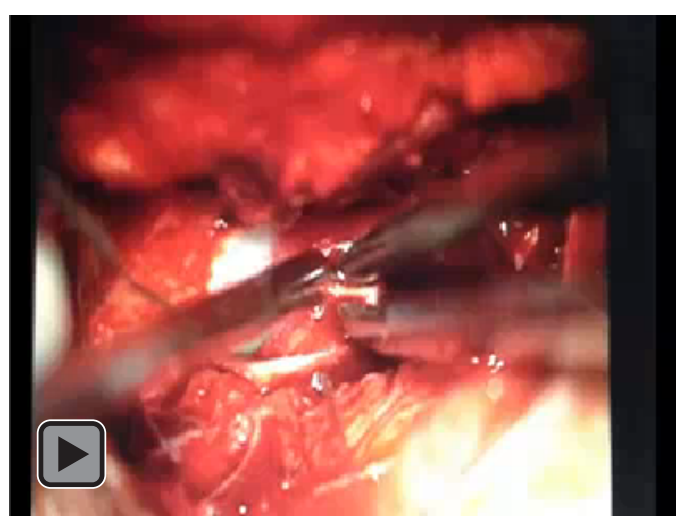

Video 2: Proceeded with the dissection of the dura and removal of scar tissue away from the injured region towards the leakage until the leak can be well visualized.

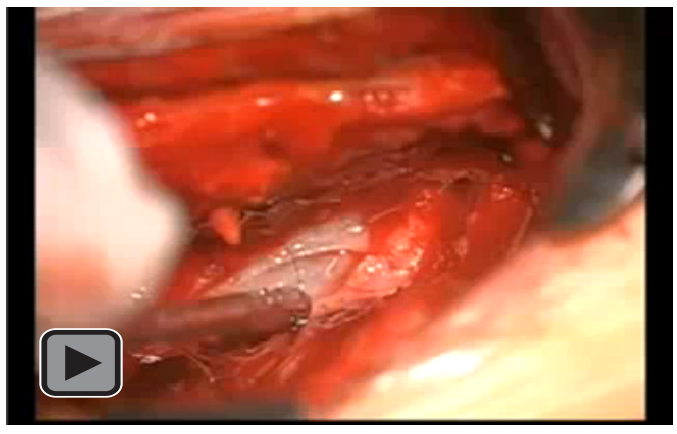

Video 3: Suture of the leak and application of a Valsalva maneuver. No further leakage can be seen. Additional repair with allogenic material and ibrin glue.

This article was originally published in a special issue, Management of Neurosurgical Complications handled by Editor. Dr. Athanasios K Petridis,

University of Schleswig Holsteinm, Germany 